\title{
Challenges in Management of the Brain-Dead Organ Donor During the COVID-19 Pandemic
}

\author{
Ioana Marina Grințescu* \\ "Carol Davila" University of Medicine and Pharmacy, Bucharest, Romania
}

Received: 19 April 2021 / Accepted: 27 April 2021

Starting in Wuhan, China [1], the infection caused by the novel SARS-CoV-2 virus became a public health issue when, due to the extreme contagiousness of this virus, a pandemic has been declared [2], putting a strain on both the global medical staff as well as the authorities in an effort to better manage an unprecedented situation in the modern era. Looking at the society we are living in, we can easily see that the COVID-19 pandemic has brought impressive social, economic, political, cultural and medical changes as well as personal ones; I believe that the perspectives and priorities of many of us have changed.

Before discussing the transplant activity, mainly the one regarding diagnosis and maintenance of the braindead organ donor patient, an activity that has been carried out for many years in Anesthesiology and Intensive Care Clinics, to which many of us are devoted, practicing it with deep respect, we need to review the daily activity. As is well known, the work effort in intensive care units is extremely demanding both mentally and physically. It involves the care of critical patients with severe decompensated pathologies, requiring maximum therapeutic management, special attention, continuous specific monitoring as well as the use of advanced medical and pharmacological techniques. The new measures and regulations, personal protective equipment, structural changes and working protocols implemented to prevent and limit COVID-19 infection, as well as the rigors imposed by the care of these patients have created additional stress for the medical staff.

In addition, I believe that social distancing has created a real communication barrier between doctors and family members, the lack of direct contact making the dialogue more difficult. All those involved in this complex activity are putting an extra effort into making the doctor-patient-family member relationship based on mutual respect and understanding.

In this special context, the National Transplant Agency of Romania issued a statement on March $16^{\text {th }}$ 2020, stipulating that the transplant activity will continue in our country while respecting the norms and provisions imposed by the competent authorities. Moreover, respecting the case definitions at the time of the intervention, specific tests both for the donor and the potential recipient should be carried out, followed by a prompt analysis which should reveal that the riskbenefit balance is clearly in favor of the patient [3]. Because "Transplant means life!".

International recommendations stipulate that in case of brain death suspicion, regardless of the infectious status, specific clinical and paraclinical examinations must be performed, depending on each case, in order to establish a definitive diagnosis (irreversible neurological injury that led to permanent loss of neurological function, including the brainstem). It is important to establish the nature of brain damage, especially given the context of hypoxic injury caused by this infection. In COVID-19 positive patients, additional measures are taken to limit the exposure of medical staff, in particular by generating aerosols during clinical examinations and performing apnea tests. Therefore, the use of HEPA respiratory filters is recommended before disconnecting the patient from the ventilator to perform the apnea tests $[4,5]$. There were some recommendations regarding diagnosis establishment based solely on brain imaging, however, the risk of producing aerosols while transporting the patients was similar [6]. 
The clinical examination should be performed by two specialists, while the apnea test should be performed by an intensive care physician and a nurse to process the arterial blood samples. COVID-19 positive patients confirmed by RT-PCR testing cannot be subjected to an organ donation/transplantation process [4,5].

Consequently, all the aforementioned aspects had an impact on the management of brain-dead potential organ donors, not in terms of diagnostic and medical management aspects, which are very well known and regulated, but in the context of the new measures imposed by this situation, the fact that transplant patients are considered "at risk" due to their immunocompromised status (requiring isolation), the intensive care beds shortage as well as the communication challenges with family members. Despite these difficulties, the Intensive Care Units throughout the country (and worldwide) $[7,8]$, accredited to carry out such activities, have made additional efforts to ensure continuity, giving people a new chance at life. Therefore, through hard work and a special devotion of the medical staff involved, in the pandemic year of 2020, a total of 190 organ transplants were performed, from 66 brain-dead donors, through multiple-organ procurement (43 donors being identified and diagnosed after the onset of the pandemic). Undoubtedly, a decline of the activity was registered compared to 2019, explained by the above mentioned aspects. These data are superposable with those reported worldwide $[7,8]$.

I believe that each of us must give ourselves a moment of reflection and introspection and try to fulfill our task to limit the harm caused by this pandemic. For my medical colleagues and for everyone working in the medical field, I wish them strength, resilience and especially balance in this period of hardship, while we continue to be guided by the principle "Primum non nocere".

\section{口CONFLICT OF INTEREST}

None to declare

\section{REFERENCES}

1. Zhu N, Zhang D, Wang W, Li X, Yang B, Song J, Zhao X, Huang B, Shi W, Lu R, Niu P. A novel coronavirus from patients with pneumonia in China, 2019. NEJM. 2020 Jan 24.

2. https://www.who.int/emergencies/diseases/novelcoronavirus-2019, Aprilie 2021

3. https://www.transplant.ro/FrontPage/2020/Comunicat\%20 ANT-COVID19-16.03.2020.pdf, Aprilie 2021

4. https://www.emoryhealthcare.org/ui/pdfs/covid/medicalprofessionals/Brain\%20Death\%20Testing\%20in\%20the\%20 Setting\%20of\%20COVID-19.pdf , accesat Aprilie 2021

5. Angelico R, et al. The COVID-19 outbreak in Italy: initial implications for organ transplantation programs. Ame Jour of Transp. 2020 Jul;20(7):1780-4.

6. Kirschen MP, McGowan N, Topjian A. Brain Death Evaluation in Children With Suspected or Confirmed Coronavirus Disease 2019. Pediatr Crit Care Med. 2021 Mar 1;22(3):318-322.

7. Migdady I, Rae-Grant A, Greer DM. Brain death evaluation during the pandemic. Neurology. 2020 Oct 13;95(15):693-4.

8. Zhang BH, Yan LN, Yang JY. Organ transplantation management in the midst of the COVID-19 outbreak: a synopsis. Hepatobiliary surgery and nutrition. $2020 \mathrm{Apr} ; 9(2): 250$. 